\begin{tabular}{|c|c|}
\hline Title & Computer generated hol ogram with characteristics of reflection: reflectance distributions and reflected images \\
\hline Author(s) & Y amaguchi, Kazuhiro; Sakamoto, Y uji \\
\hline Citation & $\begin{array}{l}\text { A pplied Optics, 48(34), H203-H211 } \\
\text { https://doi.org/10.1364/A O.48.0OH } 203\end{array}$ \\
\hline Issue Date & 2009-12-01 \\
\hline Doc URL & http:/hdl.handle.net/2115/52148 \\
\hline Rights & (c) 2009 Optical Society of A merica \\
\hline Type & article \\
\hline File Information & ao-48-34-H203.pdf \\
\hline
\end{tabular}

Instructions for use 


\title{
Computer generated hologram with characteristics of reflection: reflectance distributions and reflected images
}

\author{
Kazuhiro Yamaguchi* and Yuji Sakamoto \\ Graduate School of Information Science and Technology, Hokkaido University, \\ North 14, West 9, Kita-ku, Sapporo 060-0814, Japan \\ *Corresponding author: yamaguchi@ist.hokudai.ac.jp
}

Received 1 July 2009; revised 20 October 2009; accepted 20 October 2009; posted 21 October 2009 (Doc. ID 113656); published 10 November 2009

\begin{abstract}
This paper proposes a new computer generated hologram (CGH) method that considers the reflectance distribution on object surfaces and reflected images. The reflectance distributions are generated from phase differences determined by the shape of the object surface, which is constructed by using the Blinn and Torrance-Sparrow reflection models. Moreover, the reflected images are adapted when they are mapped onto metallic objects such as mirrors. Incorporating these two characteristics of reflection means that CGHs can express metallic objects realistically. Computer simulations and computational and optical reconstructed experiments were carried out. These results show the potential of the proposed method for showing metallic objects. (C) 2009 Optical Society of America
\end{abstract}

OCIS codes: $\quad 090.0090,090.1760$.

\section{Introduction}

The computer generated hologram $(\mathrm{CGH})$ [1] is a three-dimensional technology that can reduce the cost of generating holograms. CGHs generate hologram data by using computer simulations of optical diffraction, reflection, propagation, and interference. It embodies the principle of holography, and it can produce three-dimensional reconstructed images as in optical holography and can reconstruct virtual objects generated by computer. The holograms are generated by outputting the hologram data on some kind of display. Therefore, a CGH would be an easy way to generate holograms and reconstruct threedimensional images.

However, a lot of problems have to be overcome in order to create three-dimensional displays based on CGHs. The cost of calculating the hologram data and the poor appearance of the reconstructed images are serious issues. Many studies have tried to reduce the calculation cost. These include fast calculation

0003-6935/09/34H203-09\$15.00/0

(C) 2009 Optical Society of America methods based on parallel computing using several PCs or CPUs, graphics processing units, and other hardware [2-4]. The methods can work in real time, and they can generate CGHs or make threedimensional holo-TV images.

On the other hand, only a few studies have attempted to improve the appearance of the reconstructed images. There are a number of computer graphics $(\mathrm{CG})$ rendering techniques that can improve the appearance of displayed objects. For example, hidden surface (or line) removal is used to show occlusions between objects whose depths are different, and shading and shadowing are used to give natural luminance to three-dimensional objects. These rendering techniques can make CG models seem like real-world objects.

Some CGH researchers have used rendering to improve the appearance of reconstructed images [5], however, there has not been much research on how an object's material affects its appearance. The methods in [6-9] express various reflectance distributions, however, the incident light is limited to plane waves. If the incident light is not a plane wave or has various 
directions, which happens when there are background reflections, the reflectance distributions have to be calculated for each direction of incident light. Moreover, the above methods cannot express images reflected in a metallic or a mirror surface. Reflected images are needed to express complex natural scenes, and they should include background reflections. Background reflections can be calculated from the light from another object surface that is incident at various angles on the object surface. Thus, new approaches are needed to deal with reflectance distributions and reflected images.

The objective in our study is to express complex objects that have reflectance distributions and reflected images. The reflectance distribution is not limited by the angle of the incident light. We generate the reflectance distribution only according to the shape of the object surface, and we can reconstruct images that have reflectance distributions and reflections by using a virtual image.

To improve the quality of reconstructed images, we propose $\mathrm{CGH}$ algorithms that take into consideration reflection, i.e., reflectance distributions and reflected images. The reflectance distributions are based on the Blinn and Torrance-Sparrow reflection models in CG. We adapt these models to CGH calculations by using the phase differences of reflected light on many microfacets composing the object surface. Moreover, we adapt the reflected images to the surface by symmetric positioning of the target object and mirror. We evaluated the reflectance distributions and reflected images created by our algorithms in computer simulations and computational and optical reconstructed experiments.

\section{Calculation of Hologram Data}

Light in a CGH is considered to be a wave, so it is defined as a complex amplitude distribution. The complex amplitude distribution includes amplitude and phase values. Amplitude values mean the intensity of the light, and phase values mean the direction of the light. The hologram data are calculated from these two values.

The point light source method [10] and angular spectrum method [11] are well-known CGH calculation methods. The point light source method defines an object as a set of point light sources. The fringe pattern is calculated by superposing spherical waves at each point light source.

The angular spectrum method defines an object as a set of planar patches or images. Nonplanar objects are expressed by superposing planar patches. The object in this method is like a patch modeled object in CG, and the fringe pattern is calculated by superposing the propagations of the angular spectrum of the planar patches. Thus, the definition of objects in the angular spectrum method is more complex than in the point light source method. However, the number of point lights composing an object is generally larger than the number of planar patches. Furthermore, the angular spectrum method uses a fast
Fourier transform (FFT) to propagate each planar patch and consequently is faster than the point light method. Thus, we decided to calculate the hologram data by using the angular spectrum method.

Let us discuss the CGH calculation by referring to the basic coordinate system shown in Fig. 1. The hologram data are calculated for light propagating between the object and the hologram planes. For the input image, the complex amplitude distribution on the object plane, $g_{I}(\xi, \eta)$, is initialized by

$$
g_{I}(\xi, \eta)=A(\xi, \eta),
$$

where $\xi$ and $\eta$ are the coordinate axis on the object plane and $A(\xi, \eta)$ is the luminance of the image.

To calculate the reflection on the object surface, phase differences are added to the phase. The complex amplitude distribution after adding these phase differences, $g_{R}(\xi, \eta)$, is

$$
g_{R}(\xi, \eta)=g_{I}(\xi, \eta) \exp [-j \phi(\xi, \eta)],
$$

where $j$ denotes an imaginary number and $\phi(\xi, \eta)$ are the phase differences on the object surface.

The complex amplitude distribution on the hologram plane, $u(x, y)$, is calculated from the propagation of the reflected light from the object plane to the hologram plane. The propagation is calculated with FFT and inverse FFT [11], and the hologram data are calculated from the interference with the reference light on the hologram plane. The holograms are generated by outputting the hologram data on some sort of display device. The holograms can reconstruct images of three-dimensional objects.

\section{Expression of Reflectance Distribution}

The rendered images of a modeled object should vary according to its material. The difference in their appearances is due to differences in reflections on their surfaces. The intensity distributions of reflected light on object surfaces have very complex shapes, and the reflectance distributions are also very complex. For simplicity, the reflectance distribution in CG is defined as a synthesis of two reflections: specular reflection and diffuse reflection. Figure $\underline{2}$ shows

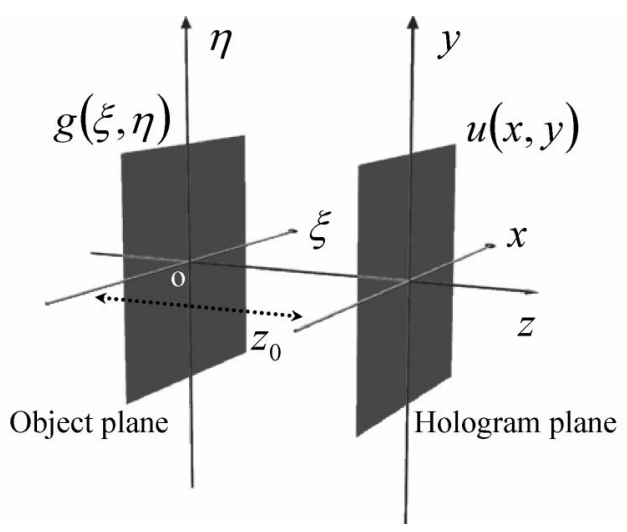

Fig. 1. Basic coordinate system. 


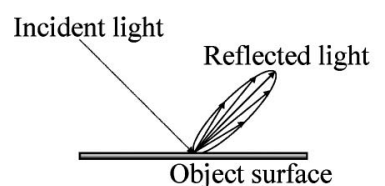

(a)

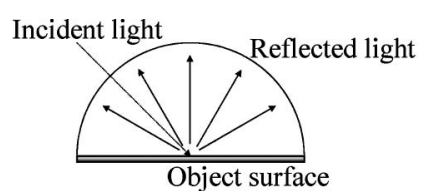

(b)
Fig. 2. Reflections on the object surface: (a) specular reflection and (b) diffuse reflection.

the reflectance distributions of (a) specular reflection and (b) diffuse reflection. In specular reflection, the reflected light on the object surface travels in a specular direction determined by the angle of the incident light and tilt angle of the object surface. Thus, an observer can see only part of the object because only part of the reflected light reaches the eye. On the other hand, in diffuse reflection, the incident light reflects in various directions off the object surface. Thus, the luminances for every reflection angle are the same, and an observer can see the whole object.

In CG, various materials can be expressed by controlling the ratio of these two reflections. The rendering methods for controlling reflections include the Phong reflection model [12], Blinn reflection model [13], Torrance--Sparrow reflection model [14], and Cook-Torrance reflection model [15].

\section{A. Conventional Methods}

As mentioned in Section 2, the reflectance distribution on the object surface can be calculated by adding phase differences to phase values. When the phase differences are 0.0 , the incident light reflects specularly on the object surface. Therefore, its reflectance distribution is a perfect specular reflection. This causes some problems. The edges of the object surface are very bright, and observers cannot see the whole object.

The random phase method can avoid these problems. The phase differences are white noise. That is, by adding white noise to the phase value, the incident light reflects in various directions. The luminance is the same in all reflected directions, and the reflectance distribution becomes a perfect diffuse reflection. To reduce the speckle noise of the random phase method, the method in Ref. [6] generates phase differences by reducing the spread of the reflected light. The method makes a nearly perfect diffuse reflection.

Other reflectance distributions can be created by controlling the ratio of specular and diffuse reflections [7-9]. In Ref. [7], the reflectance distribution is given by the Phong model, and the reflected light from the object surface is given by

$$
g_{R}(\xi, \eta)=g_{I}(\xi, \eta) s(\xi, \eta),
$$

where $s(\xi, \eta)$ is the reflectance distribution of the Phong model. Accordingly, the reflectance distribution has the characteristic of being shift invariant about the incident light direction. Thus, it is not suited for metallic objects.
The reflectance distributions in Ref. [8] are given by the Cook-Torrance model. The complex amplitude on the hologram plane from the object surface, $u(x, y)$, is calculated as

$$
u(x, y)=\hat{g}_{R}(\xi, \eta) \otimes h(\xi, \eta)
$$

where $\otimes$ denotes convolution, $\hat{g}_{R}(\xi, \eta)$ is the complex amplitude distribution of the reflected light from the object surface calculated by the random phase method, and $h(\xi, \eta)$ is the impulse response given by the Cook-Torrance model and the propagator function. These reflectance distributions can be used to express metallic surfaces. However, the calculation involves the angle between the object plane and the incident light.

The reflectance distributions in Ref. [9] depend on the size of the aperture and are calculated with the angular spectrum method. Objects are divided into many triangular patches. The bandwidth of the angular spectrum is changed by varying the width of these triangular apertures. Thus, objects appear to have various reflectance distributions depending on the widths of the triangles.

These methods assume that the incident light is a plane wave that reflects on the object surface at a certain angle. Thus, their reflectance distributions depend on the angle of the incident light. As such, they are not suited for background reflections and cannot be used to make reflected images that have various reflection angles.

\section{B. Proposed Method}

The conventional methods have limitations regarding incident light that make them unsuited for showing background reflections. Thus, we devised a new method that takes into consideration various reflectance distributions. The method is based on the Blinn and Torrance-Sparrow reflection models in CG $[13,14]$. The phase differences are calculated from the shape of the object surface generated by these reflection models. The reflectance distributions matching the shape of the object surface are generated by adding these phase differences to the phase value.

Figure 3(a) illustrates reflection on the object surface in the Blinn and Torrance-Sparrow reflection models. The surface is divided into many mirrorlike microfacets whose widths and heights are very small. The reflected light is synthesized from the light reflected from each microfacet. Each microfacet has its own tilt angle. The distribution of tilt angles is given by a facet distribution function. Note that the Blinn and Torrance-Sparrow reflection models use different facet distribution functions. In this paper, we will use the Gaussian distribution function of the Torrance-Sparrow reflection model, since they are the simplest. The Gaussian distribution function (Fig. 4) is defined as

$$
D(\theta)=\exp \left[-(\theta / m)^{2}\right]
$$




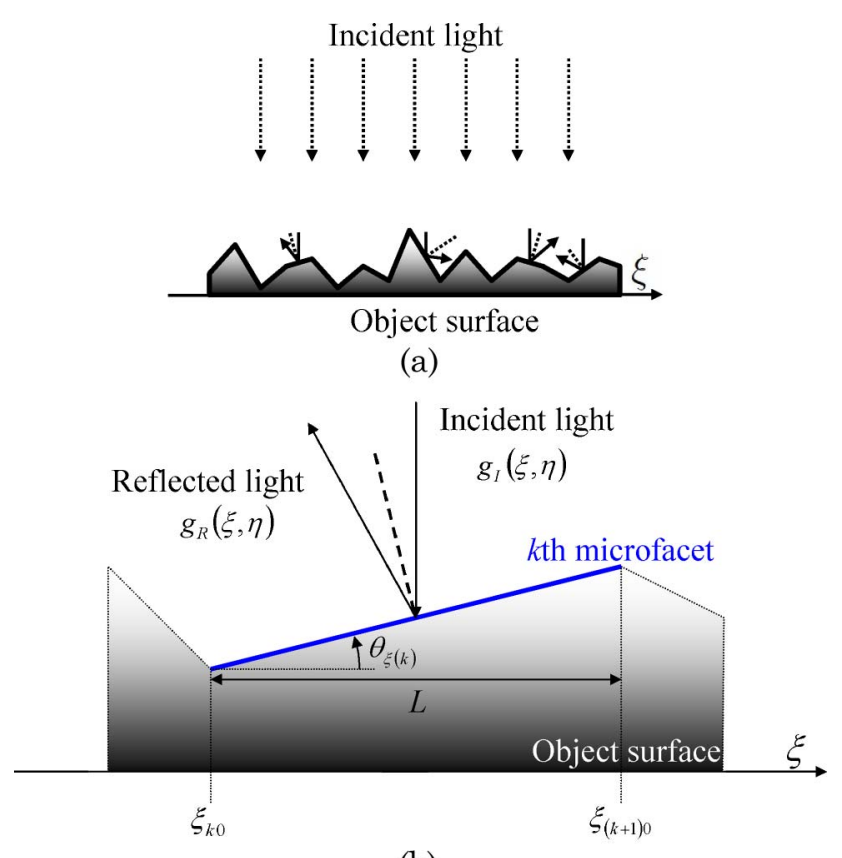

(b)

Fig. 3. (Color online) Reflection (a) on the object surface and (b) on the microfacet.

where $D(\theta)$ denotes a ratio of a direction $\theta, \theta$ is the tilt angle of a microfacet, and $m$ is the reflectance factor.

An image with a reflectance distribution is rendered in CG by multiplying $D(\theta)$ by a luminance value that depends on the position of the eye. Reflectance distributions in $\mathrm{CGH}$, however, cannot be represented in this way because the luminance of the reconstructed images varies according to the eye position. As mentioned in Section 2 , the complex amplitude distribution includes amplitude and phase values. If the amplitude, which corresponds to the luminance in CG, is changed by multiplication of $D(\theta)$ with the luminance value, the luminance of the reconstructed images will change in all directions. The luminance of a natural object will vary according to the eye position. Hence, if the luminance of the reconstructed images is the same at all eye positions, the reconstructed images will appear unnatural. Consequently, we have to adapt the reflectance distributions to CGH by using phase differences.

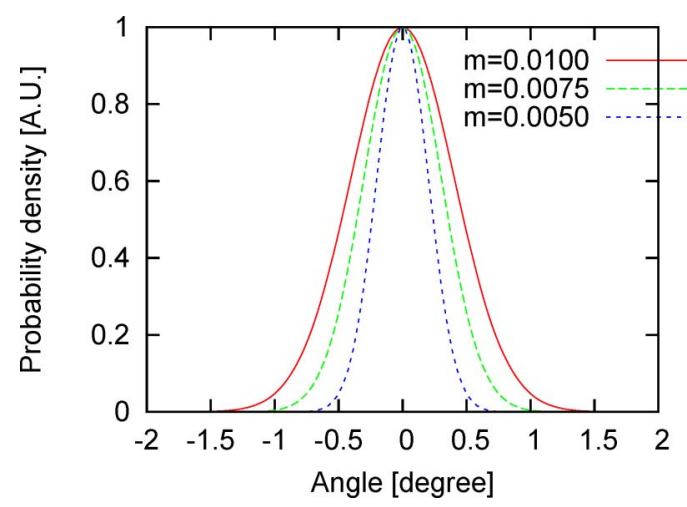

Fig. 4. (Color online) Gaussian distribution function.
Let us consider geometric reflection on the object surface. As shown in Fig. 3(a), incident light reflects in the specular direction on each microfacet. For simplicity's sake, let us consider a two-dimensional reflection on the $k$ th microfacet [Fig. 3(b)]. It is determined by the tilt angle of the microfacet and the angle of incident light. Thus, by adding phase differences calculated from the tilt angles of the microfacets to the phase, the light will reflect through the tilt angles of the microfacets. By using Eq. (2), the complex amplitude distribution of the reflected light on the object surface can be written as

$$
g_{R}(\xi, \eta)=g_{I}(\xi, \eta) \exp \left[-j 2 \phi_{M}(\xi, \eta)\right],
$$

where $\phi_{M}(\xi, \eta)$ is the phase differences of the tilt angles of the microfacets. Equation (6) is used to calculate the complex amplitude distribution of the reflected light from the phase differences $\phi_{M}(\xi, \eta)$.

The tilt angle of the $k$ th microfacet of width $L$ about the $\xi$ axis is denoted by $\theta_{\xi(k)}$. The phase differences in this microfacet, $\phi_{M(k)}\left(\xi^{\prime}\right)$, are

$$
\phi_{M(k)}(\xi)=\phi_{M(k)}\left(\xi_{k 0}\right)+\frac{2 \pi}{\lambda}\left(\xi-\xi_{k 0}\right) \tan \theta_{\xi(k)},
$$

where $\xi_{k 0}$ is the coordinate of the edge of the $k$ th microfacet and $\xi$ satisfies $\xi_{k 0} \leq \xi<\xi_{(k+1) 0}$. Thus, by summing over the number of microfacets about the $\xi$ axis, $M_{\xi}$, the phase differences of the object surface about the $\xi$ axis, $\phi_{M}(\xi)$, is

$$
\phi_{M}(\xi)=\sum_{k}^{M_{\xi}} \phi_{M(k)}(\xi),
$$

where $\phi_{M(k)}(\xi)$ is

$$
\phi_{M(k)}(\xi)=\left\{\begin{array}{c}
\phi_{M(k)}\left(\xi_{k 0}\right)+\frac{2 \pi}{\lambda}\left(\xi-\xi_{k 0}\right) \tan \theta_{\xi(k)} \\
\xi_{k 0} \leq \xi<\xi_{(k+1) 0} \\
0 \text { otherwise }
\end{array}\right.
$$

Here, $\xi_{k 0}$ denotes $\xi_{k 0}=(k-1) L$. The phase difference about the $\eta$ axis is calculated in the same way, and the phase difference about the $\xi$ and $\eta$ axes, $\phi_{M}(\xi, \eta)$, is obtained.

Note that the above calculation considers only the tilt angle about the $\xi$ or $\eta$ axis. A coordinate transformation using the rotation matrix is necessary to deal with tilt angles about the $\xi$ and $\eta$ axis at the same time.

Summarizing the above steps, the phase differences are calculated from the tilt angles of the microfacets, which follow a Gaussian distribution function. The hologram data are then calculated from these phase differences.

\section{Expression of Reflected Images}

Observers can see the environment reflected off an object surface. These "reflected images" also reveal the characteristics of specular reflection and of the 
materials. For example, observers cannot see reflected images on the surface of paper (nearly perfect diffuse reflection). On the other hand, they can see reflected images in a metal surface (nearly perfect specular reflection). Thus, to express metallic objects realistically, reflected images should be able to be seen on the object surface.

Let us consider reflection in a mirror. The reflected image depends on the positions of the mirror and the original image, as shown in Fig. 5. We assume that the viewpoint is located on the hologram plane, and the mirror and the object plane are on parallel planes. The front of the original image is directly seen from the viewpoint. The reverse side is seen through the mirror, and a reflected image is rendered on the mirror surface as if it were a texture. A CGH does not consider the reflected image because it cannot be seen directly from the viewpoint. However, as mentioned above, it is needed for expressing complex objects and scenes.

Here, in tracing a ray from the viewpoint through the mirror to an object point on the original image, the ray reflects in the specular direction. The distance between the viewpoint and the object point through the mirror is the sum of the distances between the viewpoint and the mirror and between the mirror and the object. Thus, the ray does not seem to reflect in the mirror, and it travels from the viewpoint "through" the mirror. The object gotten by tracing all the rays is called a virtual object. The virtual object and the original object are symmetric about the mirror. The propagation distance from the original image to the hologram plane through the mirror is $\Delta z+z_{0}$, and the propagation distance from the virtual object to the hologram plane is the same. Thus, the reflected image, which is symmetric to the reverse side of the original image in the mirror, appears by propagating the light wave from the virtual image to the hologram plane. The light wave propa-

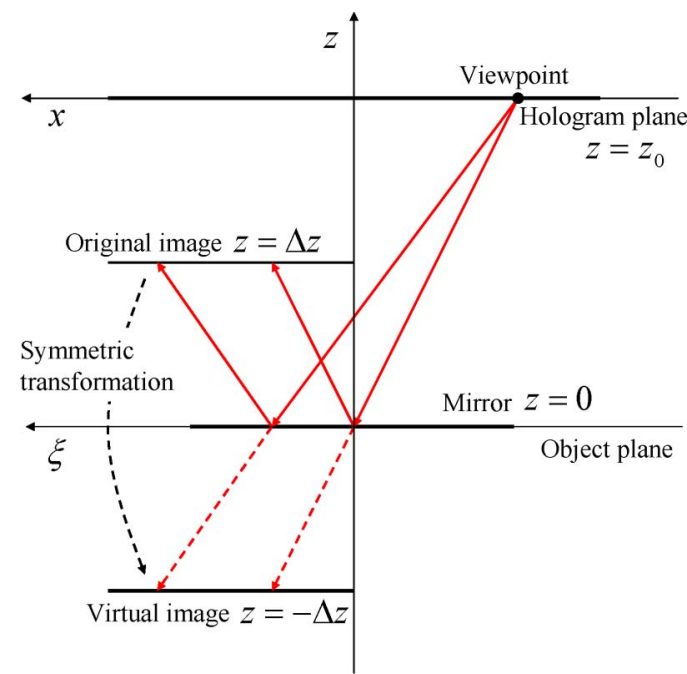

Fig. 5. (Color online) Reflection on the mirror. The virtual image is located at a symmetric position with respect to the original image in the mirror. gation from the virtual image to the hologram plane is calculated as follows:

$$
\begin{gathered}
G_{V}^{\prime}\left(f_{x}, f_{y}\right)=G_{V}\left(f_{x}, f_{y}\right) \exp \left[-j 2 \pi(-\Delta z) f_{z}\right], \\
U_{V}\left(f_{x}, f_{y}\right)=G_{V}^{\prime}\left(f_{x}, f_{y}\right) \exp \left[-j 2 \pi z_{0} f_{z}\right], \\
=G_{V}\left(f_{x}, f_{y}\right) \exp \left[-j 2 \pi\left(z_{0}-\Delta z\right) f_{z}\right],
\end{gathered}
$$

where $G_{V}\left(f_{x}, f_{y}\right)$ is the angular spectrum on the virtual image, $G_{V}^{\prime}\left(f_{x}, f_{y}\right)$ is the angular spectrum on the mirror after propagation from the virtual image, and $U_{V}\left(f_{x}, f_{y}\right)$ is the angular spectrum on the hologram plane.

We assumed that the mirror and original images are parallel. In general, they are not parallel. However, reflection in the mirror can be easily treated, and the symmetric position of an object in the mirror can be calculated in the same way as the transformation in CG. Thus, we can deal with reflected images in $\mathrm{CGH}$ simply by putting the virtual object in the place of the original object.

\section{Experiments}

To confirm the feasibility of our method, we carried out computer simulations and computational and optical reconstructed experiments.

\section{A. Computer Simulation}

The computer simulation compared reflectance distributions for different reflectance factors $m$. Here, the incident light was a coherent plane wave. Onedimensional intensity distributions of light propagating from an object surface composed of many microfacets to the hologram plane were calculated for the setup shown in Fig. 6 . The object surface had a width of about $0.051 \mathrm{~mm}$, and it had four microfacets, each with a width of about $0.013 \mathrm{~mm}$. The propagation distance, $z_{0}$, was $17 \mathrm{~mm}$. The intensity distribution on the hologram plane was calculated as a function of the angle of the origin of the object plane and each point on the hologram plane. Table 1 lists the other parameters. The incident light was a coherent wave, so the intensity distribution included speckle noise. To reduce this speckle noise,

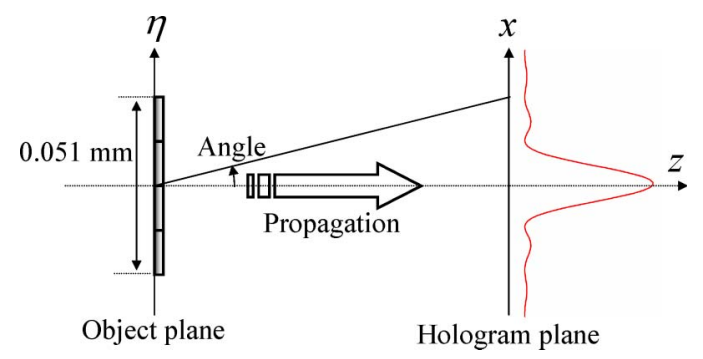

Fig. 6. (Color online) Setup of computer simulations. 
Table 1. Parameters of Computer Simulations

\begin{tabular}{ll}
\hline \multicolumn{2}{c}{ Hologram and Object Plane } \\
\hline Number of pixels & 8192 pixels \\
Size & $1.6 \mathrm{~mm}$ \\
Sampling pitch & $0.20 \mu \mathrm{m}$ \\
Propagation distance $z_{0}$ & $0.017 \mathrm{~m}$ \\
Wavelength $\lambda$ & $632 \mathrm{~nm}$ \\
Size of microfacet & 64 pixels \\
\hline
\end{tabular}

the intensity distribution was averaged over 1000 calculations.

Figure 7 shows the results plotted as intensity versus the angle shown in Fig. 6 . The reflectance factors for Figs. 7(a)-7(d) are, respectively, 0.0100, 0.0075, 0.0050 , and $0 . \overline{0025}$.

Comparing these graphs, we see that the spread of the intensity distribution depends on $m$. If $m$ is large, the distribution of the tilt angle of the microfacets grows. Thus, the incident light reflects in various directions, the spread of the intensity expands, and the reflectance distribution is one of nearly perfect diffuse reflection. On the other hand, if $m$ is small, the distribution has a small spread of a few degrees. Thus, the incident light mostly reflects in a specular direction, the spread of the intensity becomes narrow, and the reflectance distribution is one of nearly perfect specular reflection. The results of the computer simulation thus show that various reflectance distributions can be obtained by changing the reflectance factor $m$.

\section{B. Reconstruction}

We carried out computational and optical reconstructed experiments to find out whether the reflectance distributions of the reconstructed and reflected images could be obtained. For the optical reconstructed experiments, a reflective LCD (Victor Company of Japan, 4K2K LCD) was used for outputting

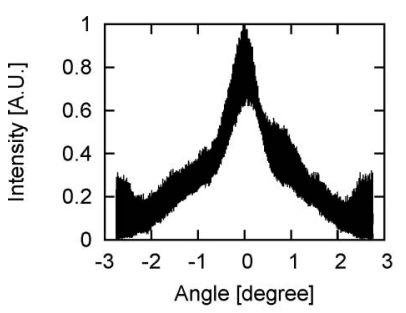

(a)

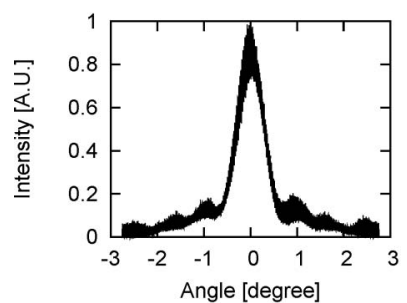

(c)

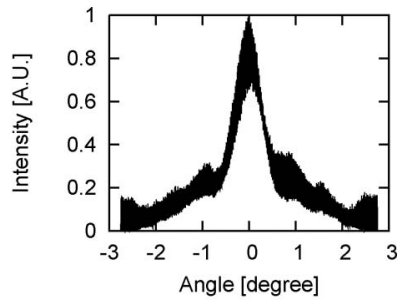

(b)

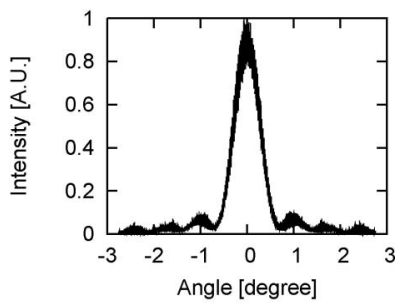

(d)
Fig. 7. Intensity distributions for different values of $m$ : (a) $m=0.0100$, (b) $m=0.0075$, (c) $m=0.0050$, (d) $m=0.0025$.
Table 2. Parameters of Optical Reconstructed Experiments

Hologram and Object Plane

\begin{tabular}{ll}
\multicolumn{2}{c}{ Hologram and Object Plane } \\
\hline Number of pixels & $2048 \times 2048$ pixels \\
Size & $19 \times 19 \mathrm{~mm}$ \\
Sampling pitch & $9.5 \times 9.5 \mu \mathrm{m}$ \\
Propagation distance $z_{0}$ & $0.15 \mathrm{~m}$ \\
Wavelength $\lambda$ & $632 \mathrm{~nm}$ \\
Size of microfacet & 2 pixels \\
\hline \multicolumn{2}{c}{ Original Image } \\
\hline Number of pixels & $1024 \times 1024$ pixels \\
Size & $0.9 \times 0.9 \mathrm{~mm}$ \\
Sampling pitch & $9.5 \times 9.5 \mu \mathrm{m}$ \\
Position $\Delta z$ & $0.03 \mathrm{~m}$ \\
\hline & \\
\hline Number of pixels & $4 \mathrm{~K} 2 \mathrm{~K} \mathrm{LCD}$ \\
Size & $38.9 \times 20.5 \mathrm{~mm}$ \\
Sampling pitch & $9.5 \times 9.5 \mu \mathrm{m}$ \\
\hline
\end{tabular}

the hologram data, and a red LED was used as the reference light. The LED was an incoherent light source, so that the speckle noise would be reduced in the reconstruction. The incident angles of the reference light were $0.0^{\circ}$ along the $x$ axis and $1.0^{\circ}$ along the $y$ axis. The other parameters are listed in Table 2 . The setup is shown in Figs. 8 and 9. In these experiments, we supposed that a mirror is located on the object plane and the observer can only see the object through the mirror. That is, the observer can only see the reverse side of the object. We also assumed that the mirror had a certain surface roughness given by the reflectance distributions, although the mirror reflected the light from the reverse side of the original image.

Figure 10 shows the block diagram for calculating the hologram data. The virtual image was calculated by using the symmetric position between the mirror and the original image. The propagation from the virtual image to the mirror was calculated by using the angular spectrum method. The incident light from the virtual image on the mirror, $g_{I}$, was obtained. To express the mirror's roughness, the phase

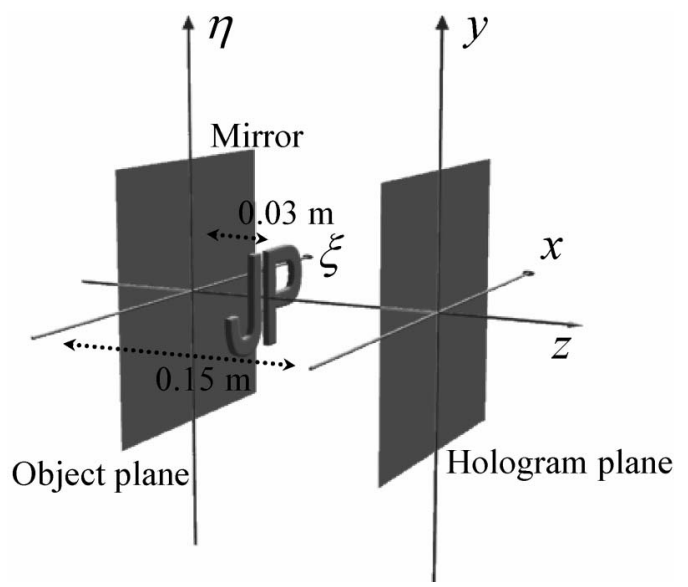

Fig. 8. Setup of computational and optical reconstructed experiments. 


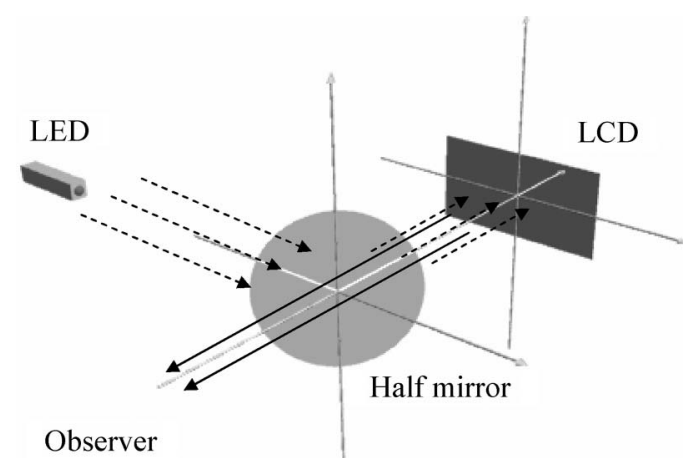

Fig. 9. Optical reconstruction system.

differences calculated with the proposed method were added to $g_{I}$ on the mirror [Eq. (6)], and the reflected light on the mirror, $g_{R}$, was calculated. The propagation from the mirror to the hologram plane was calculated, and the hologram data were obtained by calculating the interference with the reference light.

Figure 11 shows examples of phase differences on the mirror: (a) shows the amplitude of the mirror, (b) shows the phase of the random phase method, and (c) and (d) are the phases calculated by the proposed method for $m$ of 0.1000 or 0.0001 . The random phase method adds white noise, so the phases vary between pixels on the $\xi$ and $\eta$ axes. On the other hand, the phase differences given by the proposed method are continuous. Thus, the phase values given by it are also continuous.

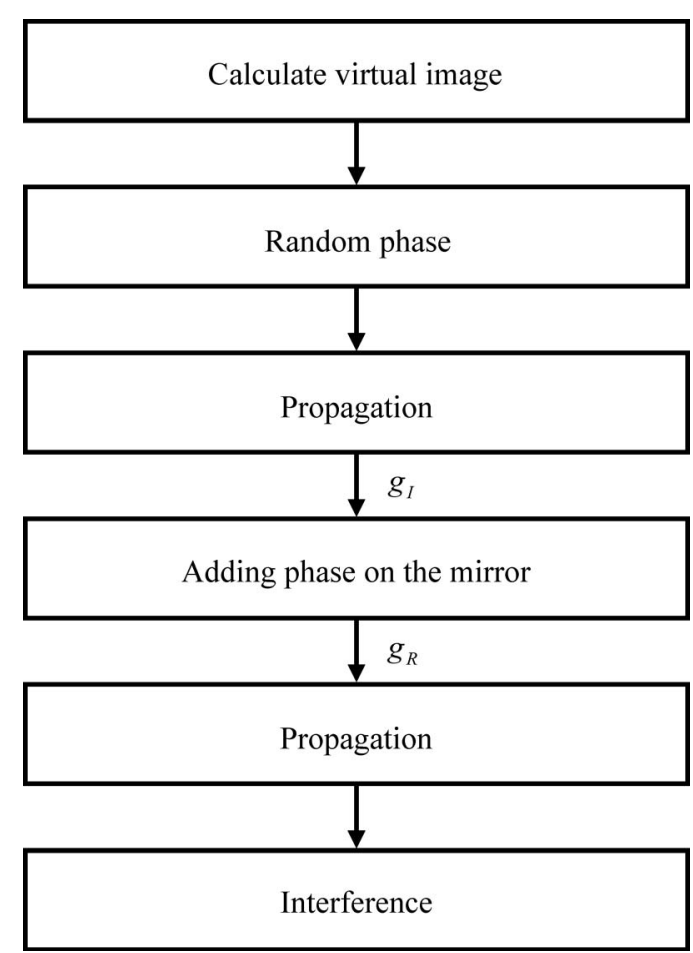

Fig. 10. Block diagram of the calculation used in computational and optical reconstructed experiments.

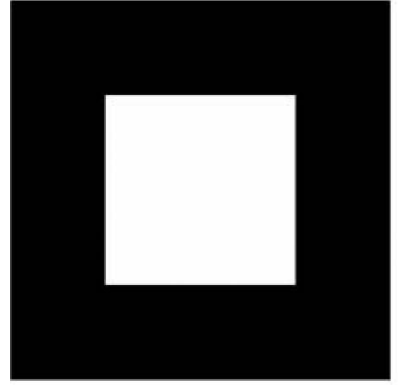

(a)

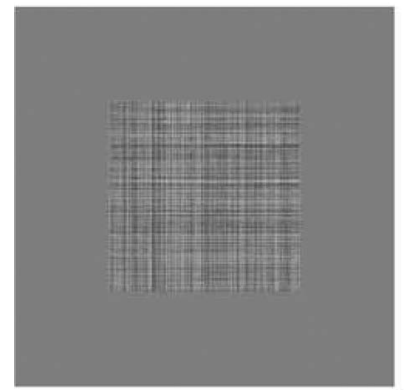

(c)

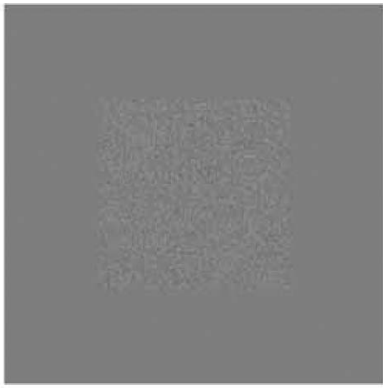

(b)

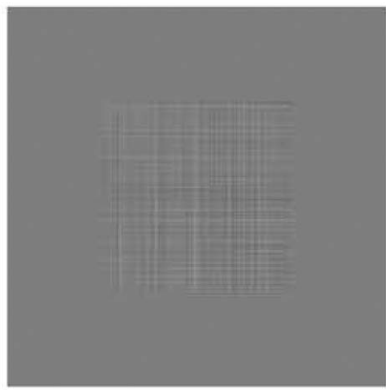

(d)
Fig. 11. Amplitude and phase distribution images: (a) amplitude image, (b) phase image with the random phase method, (c) phase image with $m=0.1000$, (d) phase image with $m=0.0001$.

Figure 12 shows the computational reconstructed images focused on the virtual image position $(z=-0.03[m])$. (a) shows the image obtained from the random phase method, (b) is the image with no phase differences, and (c)-(f) are images obtained from the proposed method with each $m$. We can see that the sharpness of the reflected images depends on the method and $m$. In Fig. 12(a), the reflectance distribution on the mirror is a perfect diffuse reflection, and the mirror has roughness. Thus, the reconstructed image has some roughness, and it appears blurry. On the other hand, in Fig. 12(b), the reflectance distribution is a perfect mirror reflection, and the mirror does not have roughness. Thus, the edges of the reflected image can be clearly seen. The reflected images obtained from the proposed method [Figs. 12(c) and 12(f)] appear to be the same. If $m$ is large, the reflectance distribution is a nearly perfect diffuse reflection, and the reflected image is the same as in the random phase method. If $m$ is small, the phase differences on the mirror become small, and the reflectance distribution is a nearly perfect mirror reflection. Thus, when $m$ is 0.0001 , the reflected image looks like the one in Fig. 12(b). If $m$ is an intermediate value, the reflected images [Figs. 12(d) and 12(e)] have more sharpness than in Fig. 12(c) and less sharpness than in Fig. 12(f).

Figure 13 shows the optical reconstructed images with the eye position at the center. These images were taken with a digital camera (Olympus, E-420) and are focused on the virtual image position $(z=-0.03 \mathrm{~m})$. They appear to be the same as the computational reconstructed images. The results of the computational and optical reconstructed 


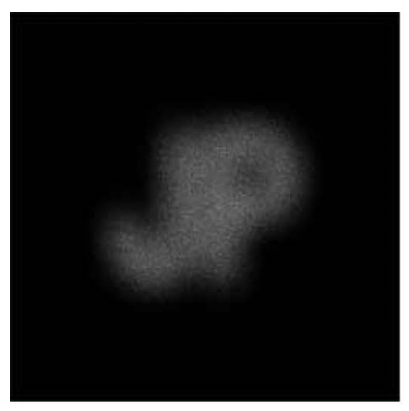

(a)

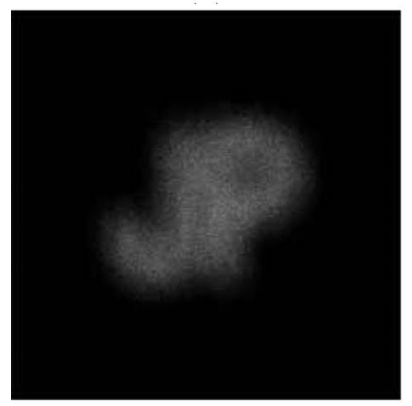

(c)

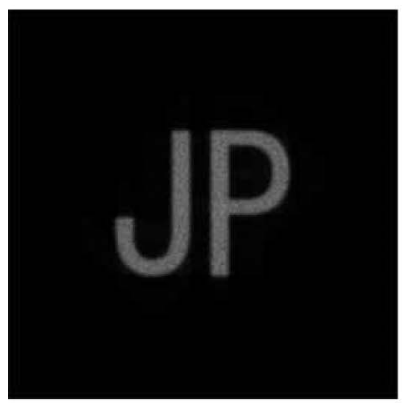

(e)

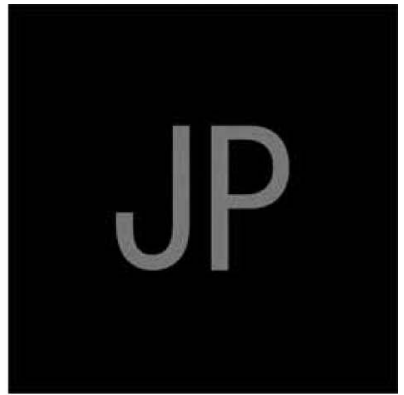

(b)

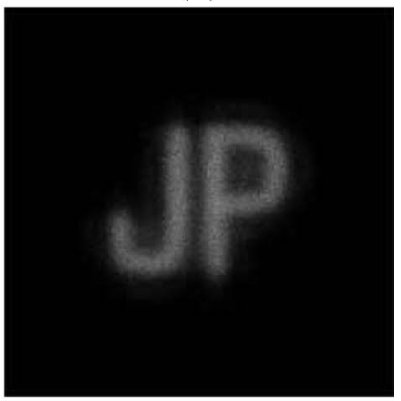

(d)

(e)

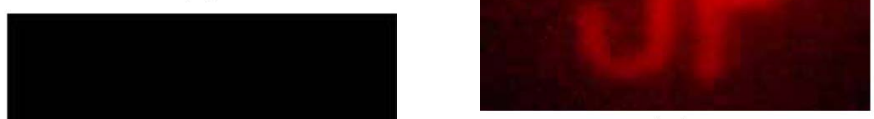

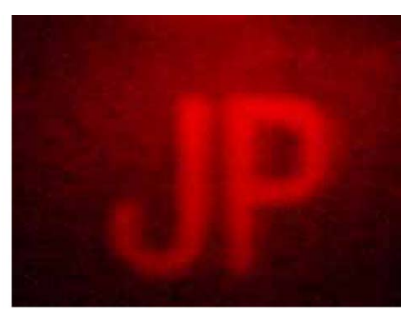

(b)

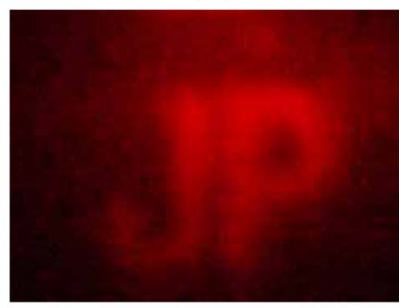

(d)

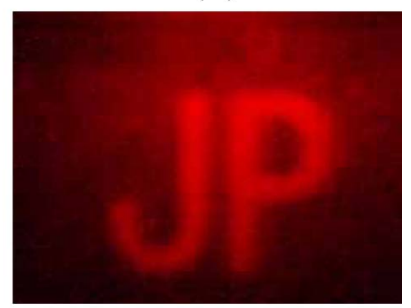

(f)
Fig. 13. (Color online) Optical reconstructed images with (a) the random phase method, (b) no phase differences, (c) $m=0.1000$, (d) $m=0.0100$, (e) $m=0.0010$, (f) $m=0.0001$.

varying size. To express such real-world objects, we will need to consider the size and distribution of microfacets.

\section{Conclusion}

We proposed a computer generated hologram method that takes into account reflectance distributions and reflected images. The method adapts the Blinn and Torrance-Sparrow reflection models to the CGH calculation of the phase difference. Reflectance distributions are given by changing the tilt angles of mirrorlike microfacets of the object surface. Moreover, metallic objects can be expressed by considering reflected images, i.e., images reflected through a mirror.

The computer simulations, computational and optical reconstructed experiments, showed that the reflectance distributions vary according to the phase differences following the shape of the object surface and not according to the position of the incident light. The reflected images are calculated from the propagation of virtual images at a position symmetric to that of original images in a mirror. We achieved our objectives of making reflectance distributions that do not vary with the position of the incident light and expressing reflected images. In the future, we should develop a way to vary the size of the microfacet to make reflectance distributions that can express more complex objects. 
The authors thank members of Victor Company of Japan, Kurihama R\&D Center, for letting us use their LCD.

\section{References}

1. J. W. Goodman, Introduction to Fourier Optics (McGraw-Hill, 2nd ed. (1996).

2. H. Kang, T. Yamaguchi, H. Yoshikawa, S.-C. Kim, and E.-S. Kim, "Acceleration method of computing a compensated phase-added stereogram on a graphic processing unit," Appl. Opt. 47, 5784-5789 (2008).

3. T. Yamaguchi, T. Fujii, and H. Yoshikawa, "Fast calculation method for computer-generated cylindrical holograms," Appl. Opt. 47, D63-D70 (2008).

4. Y. Ichihashi, H. Nakayama, T. Ito, N. Masuda, T. Shimobaba, A. Shiraki, and T. Sugie, "HORN-6 special-purpose clustered computing system for electroholography," Opt. Express 17, 13895-13903 (2009).

5. K. Matsushima, "Computer-generated holograms for threedimensional surface objects with shade and texture," Appl. Opt. 44, 4607-4614 (2005).

6. R. Bräuer, F. Wyrowski, and O. Bryngdahl, "Diffusers in digital holography," J. Opt. Soc. Am. A 8, 572-578 (1991).

7. Y. Sakamoto and Y. Yamashita, "An algorithm for object-light calculation considering reflectance distribution for computer- generated holograms," J. Inst. Image Information Television Engineers 56, 611-616 (2002) (in Japanese).

8. Y. Sakamoto and A. Tsuruno, "A representation method for object surface glossiness in computer-generated hologram," IEICE Trans. Information Syst. 2 J88-D-2, 2046-2053 (2005).

9. H. Kim, J. Hahn, and B. Lee, "Mathematical modeling of triangle-mesh-modeled three-dimensional surface objects for digital holography," Appl. Opt. 47, D117-D127 (2008).

10. J. P. Waters, "Holographic image synthesis utilizing theoretical methods," Appl. Phys. Lett. 9, 405-407 (1966).

11. K. Matsushima, H. Schimmel, and F. Wyrowski, "Fast calculation method for optical diffraction on tilted planes by use of the angular spectrum of plane waves," J. Opt. Soc. Am. A 20, 1755-1762 (2003).

12. B. T. Phong, "Illumination for computer generated pictures," Commun. ACM 18, 311-317 (1975).

13. J. F. Blinn, "Models of light reflection for computer synthesized pictures," SIGGRAPH Comput. Graph. 11, 192-198 (1977).

14. K. E. Torrance and E. M. Sparrow, "Theory for off-specular reflection from roughened surfaces," J. Opt. Soc. Am. 57, 1105-1112 (1967).

15. R. L. Cook and K. E. Torrance, "A reflectance model for computer graphics,” ACM Trans. Graph. 1, 7-24 (1982). 